\title{
Partial $f$-Sum Rules for Rotator Strengths
}

\author{
Heinz Dieter Rudolph \\ Physikalisches Institut der Universität Freiburg i. Br. \\ (Z. Naturforsch. 23 a, 1020-1023 [1968] ; received 25 March 1968)
}

\begin{abstract}
In extension of a previous paper partial $f$-sum rules are derived for the rotator strengths of a freely rotating asymmetric top molecule whose permanent dipole moment interacts with the radiation field. In either of the two relations given the sums run over only part of the possible rotational transitions which start from any particular level: (1) $J \rightarrow J, J+1$; (2) $J \rightarrow J, J-1$. The two relations express stronger sum rules than the one reported formerly which may be shown to be dependent on those of the present paper. A short outline is given on how the "rotator strength" of this paper may be connected with the "oscillator strength for a rotational transition" of dispersion theory.
\end{abstract}

In a recent paper (hereinafter referred to as I) it has been shown that certain quantities termed "rotator strengths" for the rotational energy transitions of a molecule obey a sum rule analogous to the familiar Kunn-Thomas-Reiche $f$-sum rule ${ }^{2,3}$ for the oscillator strengths of the electrons in an atom. Eq. (I.23) states that the sum of the rotator strengths for all possible rotational energy transitions $J \rightarrow J, J \pm 1$ induced by a body-fixed dipole moment component $\mu_{g}$ of the molecule and starting from any particular rotational level $J \tau$ equals one.

Using a method first applied by Wigner ${ }^{4-6}$ to the original $f$-sum rule it is possible to show that simple expressions exist also for sums over rotator strengths that extend over part of the admissible rotational transitions, e.g. $J \rightarrow J, J+1$ or $J \rightarrow J$, $J-1$. These partial sums are not constants, in this respect unlike those of WIGNER. They are, however, easily evaluable functions of the rotational state $J \tau$. This difference may by reference to I be traced back to the fact that the sum rules for the rotator strengths must be developped from a commutation relation between two non-conjugate variables whereas the original $f$-sum rule for oscillator strengths follows from a commutation relation between the conjugate position and momentum coordinates of the electrons in the atom.

\section{Molecular Rotator and f-Sum Rule}

Let the rotating molecule be approximated by a rigid asymmetric top and its motion be described by the direction cosine operators $\Phi_{F g}$ between a

1 H. D. Rudolph, Z. Phys. 211, 419 [1968].

2 W. Kunn, Z. Phys. 33, 408 [1925].

3 F. Reiche and W. Thomas, Z. Phys. 34, 510 [1925].

4 E. WIGNER, Phys. Z. 32, 450 [1931]; see also ${ }^{5}, 6$.

5 J. G. KIrkwood, Phys. Z. 33, 521 [1932]. space-fixed frame of reference $F=X, Y, Z$ and the body-fixed principal axes of inertia $g=x, y, z$. The principal moments of inertia will be denoted by $I_{g}$, $g=x, y, z$. Rotational energy transitions are produced by the interaction of the radiation field with the permanent electrical dipole moment of the molecule with the (constant) components $\mu_{g}$. It has been shown in I that the commutation relation between the non-conjugate variables $\Phi_{F g}$ and $\dot{\Phi}_{F g}$

$$
\frac{i}{\hbar}\left(\dot{\Phi}_{F g} \Phi_{F g}-\Phi_{F g} \dot{\Phi}_{F g}\right)=\frac{\Phi_{F g^{\prime \prime}}^{2}}{I_{g^{\prime}}}+\frac{\Phi_{F g^{\prime}}^{2}}{I_{g^{\prime \prime}}} \begin{aligned}
& F=X, Y, Z \\
& g=x, y, z
\end{aligned}
$$

yields, for an eigenstate $\mid J \tau M$ ) with the rotational energy eigenvalue $W_{J \tau}$, an equivalent of what has earlier been called the "general $f$-sum rule ${ }^{7}$ "

$$
\begin{aligned}
& \sum_{J^{\prime} \tau^{\prime} M^{\prime}}\left(W_{J^{\prime} \tau^{\prime}}-W_{J \tau}\right)\left|\left(J^{\prime} \tau^{\prime} M^{\prime}\left|\Phi_{F g}\right| J \tau M\right)\right|^{2} \\
& \quad=G^{\prime}\left(J \tau M\left|\Phi_{F g^{\prime \prime}}^{2}\right| J \tau M\right)+G^{\prime \prime}\left(J \tau M\left|\Phi_{F g^{\prime}}^{2}\right| J \tau M\right)
\end{aligned}
$$

where "rotational constants" $G=\hbar^{2} / 2 I_{g}, G^{\prime}$ and $G^{\prime \prime}$ accordingly, have been introduced. The individual terms of the left-hand sum are proportional to the frequency times the dipole moment matrix element squared for all possible transitions which start from the particular level $J \tau M$ considered and can be induced by the dipole moment component $\mu_{g}$ under the influence of radiation polarized in $F$. direction. It is this equation (and not the one summed over $F$ and/or $M$ to make it equal a constant as in I which must be used in the present paper to derive the partial $f$-sum rules.

${ }^{6}$ J. C. Slater, Quantum Theory of Atomic Structure, Vol. II, McGraw-Hill Book Co., New York 1960, p. 227.

7 H. A. Bethe and E. E. Salpeter, Quantum Mechanics of One- and Two-Electron Systems, in Encyclopedia of Physics, S. FLügGe ed., Vol. XXXV, Springer-Verlag, Berlin 1957, p. 342. 
Each matrix element $\left(J^{\prime} \tau^{\prime} M^{\prime}\left|\Phi_{F g}\right| J \tau M\right)$ factors ${ }^{8}$ into a $J$-, $J \tau$-, and $J M$-dependent term. The $M$-dependence of the sums $\sum_{M}{ }^{\prime}\left|\left(J^{\prime} \tau^{\prime} M^{\prime}\left|\Phi_{F g}\right| J \tau M\right)\right|^{2}$ may hence be shown explicitly after the $M$-independent factor has been expressed with the aid of the "line strength"

$$
S^{g}\left(J^{\prime} \tau^{\prime}, J \tau\right)=3 \sum_{M M}\left|\left(J^{\prime} \tau^{\prime} M^{\prime}\left|\Phi_{F g}\right| J \tau M\right)\right|^{2}
$$

[Eq. (I.11)], e. g. for $F=Z$ and any $g$

$$
\begin{gathered}
\left|\left(J+1 \tau^{\prime} M\left|\Phi_{Z g}\right| J \tau M\right)\right|^{2} \\
=\frac{(J+1)^{2}-M^{2}}{(2 J+1)(J+1)(2 J+3)} S^{g}\left(J+1 \tau^{\prime}, J \tau\right), \\
\left|\left(J \tau^{\prime} M\left|\Phi_{Z g}\right| J \tau M\right)\right|^{2} \\
=\frac{M^{2}}{J(2 J+1)(J+1)} S^{g}\left(J \tau^{\prime}, J \tau\right), \\
\left|\left(J-1 \tau^{\prime} M\left|\Phi_{Z g}\right| J \tau M\right)\right|^{2} \\
=\frac{J^{2}-M^{2}}{(2 J-1) J(2 J+1)} S^{g}\left(J-1 \tau^{\prime}, J \tau\right)
\end{gathered}
$$

where the sum over $M^{\prime}$ reduces to one term because $\Phi_{Z g}$ is diagonal in $M$. Either by direct calculation or by the application of the Wigner-Eckart theorem the diagonal matrix elements of the direction cosines squared may be shown to be (I, footnote ${ }^{17}$; here $F=Z$, any $g$ )

$$
\begin{aligned}
& \left(J \tau M\left|\Phi_{Z g}^{2}\right| J \tau M\right)= \\
& \quad=\frac{1}{3}+\frac{2\left[3\left\langle P_{g}^{2}\right\rangle_{J \tau}-J(J+1)\right]}{3(2 J-1) J(J+1)(2 J+3)}\left(3 M^{2}-J(J+1)\right)
\end{aligned}
$$

where we have written the symbol $\left\langle P_{g}{ }^{2}\right\rangle_{J \tau}$ in place of the dimensionless quantity $\left(J \tau\left|P_{g}{ }^{2}\right| J \tau\right) / \hbar^{2}$ in order to avoid a cumbersome actor of $1 / \hbar^{2}$ in subsequent equations. Substituting (1) and (2) into (I.18) one obtains the following equation which is independent of $F$, and holds for any $g$ and any initial level $J \tau M$

$$
\begin{aligned}
& \frac{(J+1)^{2}-M^{2}}{(J+1)(2 J+3)} \sum_{\tau^{\prime}} f^{g}\left(J+1 \tau^{\prime}, J \tau\right)+\frac{M^{2}}{J(J+1)} \sum_{\tau^{\prime}} f^{g}\left(J \tau^{\prime}, J \tau\right)+\frac{J^{2}-M^{2}}{(2 J-1) J} \sum_{\tau^{\prime}} f^{g}\left(J-1 \tau^{\prime}, J \tau\right) \\
& \quad=\frac{1}{3}-2\left[\frac{J(J+1)}{3}-\frac{G^{\prime}\left\langle P_{g^{\prime \prime}}^{2}\right\rangle J_{\tau}+G^{\prime \prime}\left\langle P_{g^{\prime}}^{2}\right\rangle J \tau}{G^{\prime}+G^{\prime \prime}}\right] \frac{3 M^{2}-J(J+1)}{(2 J-1) J(J+1)(2 J+3)} .
\end{aligned}
$$

The left-hand member of the equation consists of the three partial sums for $J \rightarrow J+1, J$, and $J-1$ over the rotator strengths $f$ as defined in (I.23)

$$
f^{g}\left(J^{\prime} \tau^{\prime}, J \tau\right) \equiv \frac{W_{J^{\prime} \tau^{\prime}}-W_{J \tau}}{G^{\prime}+G^{\prime \prime}} \frac{S^{g}\left(J^{\prime} \tau^{\prime}, J \tau\right)}{2 J+1}=\frac{8 \pi^{2}}{h\left(1 / I_{g^{\prime}}+1 / I_{g^{\prime \prime}}\right)} v\left(J^{\prime} \tau^{\prime}, J \tau\right) \frac{S^{g}\left(J^{\prime} \tau^{\prime}, J \tau\right)}{2 J+1}
$$

$G, G^{\prime}, G^{\prime \prime}$ corresponding to $g, g^{\prime}, g^{\prime \prime}=x, y, z$; any order.

Note that the "sum rule for rotator strengths", Eq. (I.23), may be written

$$
\sum_{\tau^{\prime}} f^{g}\left(J+1 \tau^{\prime}, J \tau\right)+\sum_{\tau^{\prime}} f^{g}\left(J \tau^{\prime}, J \tau\right)+\sum_{\tau^{\prime}} f^{g}\left(J-1 \tau^{\prime}, J \tau\right)=1
$$

and could have been derived by summing (3) from $M=-J$ to $M=+J^{9}$.

\section{Partial f-Sum Rules}

The quantum number $M$ in (3) can be assigned any allowed numerical value, the resulting equation is then an $M$-independent linear relation between the three partial sums. Different equations so obtained are, however, by no means independent. In fact, Eq. (5) plus any two Eqs. (3) with different values $M$ may be shown to constitute a system of dependent equations whose determinant vanishes. Convenient expressions result when we choose two different linear combinations of Eqs. (3) for fixed $M$ either containing only two of the three partial sums ${ }^{10}$. After multiplication by a common factor we have

$$
\begin{aligned}
\sum_{\tau^{\prime}}\left(W_{J+1 \tau^{\prime}}\right. & \left.-W_{J \tau}\right) S^{g}\left(J+1 \tau^{\prime}, J \tau\right)+\frac{J(2 J+3)}{2 J+1} \sum_{\tau^{\prime}}\left(W_{J \tau^{\prime}}-W_{\tau_{\tau}}\right) S^{g}\left(J \tau^{\prime}, J \tau\right) \\
& =(J+1)\left(G^{\prime}+G^{\prime \prime}\right)+2\left(G^{\prime}\left\langle P_{g^{\prime \prime}}^{2}\right\rangle_{J \tau}+G^{\prime \prime}\left\langle P_{g^{\prime}}^{2}\right\rangle_{J \tau}\right) \\
& =(J+1)(2 J+1)\left(G^{\prime}+G^{\prime \prime}\right)-2 W_{J \tau}+2\left(G-G^{\prime}-G^{\prime \prime}\right)\left\langle P_{g}{ }^{2}\right\rangle_{J \tau},
\end{aligned}
$$

8 P. C. Cross, R. M. Hainer, and G. W. King, J. Chem. Phys. 12,210 [1944]. See also ref. ${ }^{9 \mathrm{a}}$ to ${ }^{9 \mathrm{~d}}$ of $\mathrm{I}$.

9 Since $\sum_{-J}^{+J} M^{2}=\frac{1}{3} J(J+1)(2 J+1)$ the factor multiplying the square bracket vanishes upon summation.
10 To obtain (6 a) take Eq. (3) for $M=J$. To obtain (6b) subtract $(J+1)^{2}$ times Eq. (3) for $M=J$ from $(2 J+1)$ times Eq. (3) for $M=0$. 


$$
\begin{gathered}
-\frac{(2 J-1)(J+1)}{2 J+1} \sum_{\tau^{\prime}}\left(W_{J \tau^{\prime}}-W_{J \tau}\right) S^{g}\left(J \tau^{\prime}, J \tau\right)+\sum_{\tau^{\prime}}\left(W_{J-1 \tau^{\prime}}-W_{J \tau}\right) S^{g}\left(J-1 \tau^{\prime}, J \tau\right) \\
=J\left(G^{\prime}+G^{\prime \prime}\right)-2\left(G^{\prime}\left\langle P_{g^{\prime \prime}}^{2}\right\rangle_{J \tau}+G^{\prime \prime}\left\langle P_{g^{\prime}}^{2}\right\rangle_{J \tau}\right) \\
=-J(2 J+1)\left(G^{\prime}+G^{\prime \prime}\right)+2 W_{J_{\tau}}-2\left(G-G^{\prime}-G^{\prime \prime}\right)\left\langle P_{g}^{2}\right\rangle_{J \tau} .
\end{gathered}
$$

The last members of Eqs. (6a) and (6b) give alternative forms. In writing them down use has been made of the following expressions for the eigenvalues of the square of the angular momentum and the rotational energy

$$
\begin{aligned}
& \left\langle P_{g}^{2}\right\rangle_{J \tau}+\left\langle P_{g^{\prime}}^{2}\right\rangle_{J_{\tau}}+\left\langle P_{g^{\prime \prime}}^{2}\right\rangle_{J_{\tau}}=J(J+1), \\
& G\left\langle P_{g}^{2}\right\rangle_{J \tau}+G^{\prime}\left\langle P_{g^{\prime}}^{2}\right\rangle_{J \tau}+G^{\prime \prime}\left\langle P_{g^{\prime \prime}}^{2}\right\rangle_{J \tau}=W_{J_{\tau}} .
\end{aligned}
$$

Equations (6a) and $(6 \mathrm{~b})$ together [after division by $\left.(2 J+1)\left(G^{\prime}+G^{\prime \prime}\right)\right]$ may be considered to express stronger "partial $f$-sum rules" than the simple " $f$-sum rule" (5) derived in I which is easily seen to follow from (6a) and (6b) by addition.

\section{Rules in Reduced Form}

In rotational spectroscopy it is convenient to reduce the eigenvalue problem to dimensionless form by setting $A=+1, B=\varkappa, C=-1$ where $\varkappa$ is the inertial asymmetry parameter of the problem. $A, B$, and $C$ are conventionally identified with $G, G^{\prime}, G^{\prime \prime}$, defined above, according to $A \geqq B \geqq C$.

$$
\varkappa \equiv(2 B-A-C) /(A-C),(-1 \leqq \varkappa \leqq+1) .
$$

The "reduced" energy is from (7)

$$
E_{J \tau}(\varkappa)=\left\langle P_{a}^{2}\right\rangle_{J \tau}+\varkappa\left\langle P_{b}^{2}\right\rangle_{J \tau}-\left\langle P_{c}^{2}\right\rangle_{J \tau}
$$

where $a, b$, and $c$ have been correspondingly identified with the directions $g, g^{\prime}, g^{\prime \prime}$. Hence

$$
W_{J \tau}=\frac{1}{2}(A+C) J(J+1)+\frac{1}{2}(A-C) E_{J \tau}(\varkappa) .
$$

The quantities $E_{J \tau}$ as well as $\left\langle P_{g}{ }^{2}\right\rangle_{J \tau}$ and $S^{g}\left(J^{\prime} \tau^{\prime}, J \tau\right)$ have the advantage that they depend for arbitrary rotational constants of the molecule on the inertial asymmetry parameter $\varkappa$ only, a fact that has rendered them accessible to easy tabulation. Upon introduction of the reduced energy Eqs. (6a) and (6b) finally become

$$
\begin{aligned}
& \sum_{\tau^{\prime}}\left(E_{J+1 \tau^{\prime}}-E_{J \tau}\right) S^{g}\left(J+1 \tau^{\prime}, J \tau\right)+\frac{J(2 J+3)}{2 J+1} \sum_{\tau^{\prime}}\left(E_{J \tau^{\prime}}-E_{J \tau}\right) S^{g}\left(J \tau^{\prime}, J \tau\right) \\
& =\left\{\begin{array}{rlrl}
(\varkappa-1)(J+1)+2 \varkappa\left\langle P_{c}{ }^{2}\right\rangle_{J \tau}-2\left\langle P_{b}{ }^{2}\right\rangle_{J \tau} & =(\varkappa-1)(J+1)(2 J+1) & -2 E_{J \tau}+2(2-\varkappa)\left\langle P_{a}{ }^{2}\right\rangle_{J \tau} & \text { for } g=a, \\
-2\left\langle P_{a}{ }^{2}\right\rangle_{J \tau}+2\left\langle P_{c}{ }^{2}\right\rangle_{J \tau} & = & -2 E_{J \tau}+2 \varkappa\left\langle P_{b}{ }^{2}\right\rangle_{J \tau} & \text { for } g=b, \\
(\varkappa+1)(J+1)+2\left\langle P_{b}{ }^{2}\right\rangle_{J \tau}+2 \varkappa\left\langle P_{a}^{2}\right\rangle_{J \tau} & =(\varkappa+1)(J+1)(2 J+1)-2 E_{J \tau}-2(2+\varkappa)\left\langle P_{c}{ }^{2}\right\rangle_{J \tau} \text { for } g=c ;
\end{array}\right. \\
& -\frac{(2 J-1)(J+1)}{2 J+1} \sum_{\tau^{\prime}}\left(E_{J \tau^{\prime}}-E_{J \tau}\right) S^{g}\left(J \tau^{\prime}, J \tau\right)+\sum_{\tau^{\prime}}\left(E_{J-1 \tau^{\prime}}-E_{J \tau}\right) S^{g}\left(J-1 \tau^{\prime}, J \tau\right)
\end{aligned}
$$

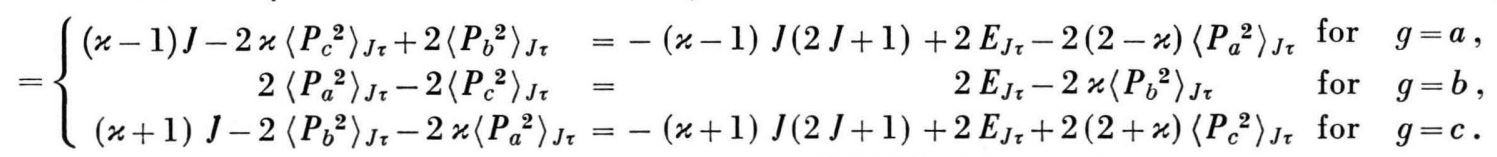

Eqs. (8a) and (8b) should be compared with Eq. (I.24). The latter is seen to be the sum $(8 a)+(8 b)$ separately for $g=a, b, c$. We remark in conclusion that summing $(6 \mathrm{a}, \mathrm{b})$ or $(8 \mathrm{a}, \mathrm{b})$ over $g$ leads to relations which do not contain the expectation values $\left\langle\boldsymbol{P}_{g}{ }^{2}\right\rangle_{J \tau}$

$$
\begin{aligned}
& \sum_{g}(6 \mathrm{a})=2(J+1)^{2}\left(G+G^{\prime}+G^{\prime \prime}\right)-2 W_{J \tau} ; \\
& \sum_{g}(6 \mathrm{~b})=-2 J^{2}\left(G+G^{\prime}+G^{\prime \prime}\right)+2 W_{J_{\tau}}
\end{aligned}
$$

and correspondingly

$$
\begin{aligned}
& \sum_{g}(8 \mathrm{a})=2 \varkappa(J+1)^{2}-2 E_{J \tau} ; \\
& \sum_{g}(8 \mathrm{~b})=-2 \varkappa J^{2}+2 E_{J \tau} .
\end{aligned}
$$

\section{A Dispersion Theory Model}

The definition of "rotator strength" (4) as used in I and this paper was based on the complete analogy of Eqs. (I.23) and (I.5). We shall in conclusion try to connect this definition with that of the "strength" of any meaningful oscillator model in the framework of dispersion theory. In dispersion theory the electronic transitions of an atom are represented by a multitude of classical isotropic oscillators each with electrical charge $e$ and mass $m_{0}$ and a resonance frequency which equals one of the possible transition frequencies. The substitute oscillators are polarizable by the electrical vector of the 
light wave, and each oscillator can be shown to contribute to the true polarizability of matter to the extent given by its oscillator strength. Dispersion theory for a gas of rotating molecules with dipole moment components (I.9) follows quite closely along the lines given in the textbooks ${ }^{11-13}$ and leads to the same result. After summation over the degenerate $M$-sublevels the (isotropic) polarizability at frequency $v$ of a gas of rigid rotor molecules with dipole moment $\mu_{g}$ in the rotational state $J \tau$ is

$$
\begin{aligned}
\alpha^{g}(J \tau) & \equiv\left(J \tau\left|\alpha^{g}\right| J \tau\right) \\
& =\frac{2 \mu g^{2}}{3 h} \sum_{J^{\prime} \tau^{\prime}} \frac{v\left(J^{\prime} \tau^{\prime}, J \tau\right) S g\left(J^{\prime} \tau^{\prime}, J \tau\right)}{(2 J+1)\left(v^{2}\left(J^{\prime} \tau^{\prime}, J \tau\right)-v^{2}\right)} .
\end{aligned}
$$

To the macroscopic polarizability each state contributes according to its population. It is now possible to construct a model such that the polarizability (11) equals the sum over the polarizabilities of classical rotatory oscillators each weighted by its rotator strength as defined in (4) :

$$
\alpha^{g}(J \tau)=\sum_{J^{\prime} \tau^{\prime}} f^{g}\left(J^{\prime} \tau^{\prime}, J \tau\right) \cdot \alpha_{\mathrm{cl}}^{g}\left(J^{\prime} \tau^{\prime}, J \tau\right) .
$$

To this effect let each rotational transition of a molecule be represented by a rod-shaped rotatory oscillator with fixed turning point and with the rod pointing into an arbitrary but fixed zero-direction, capable of small-amplitude rotatory vibrations about any axis through its center perpendicular to the zero-direction of the rod. The resonance frequency equals the rotational transition frequency which it represents. The rod carries the dipole moment $\mu_{g}$ upon which the passing light wave of frequency $v$ exerts a torque exciting forced oscillations. The reciprocal moment of inertia $1 / I$ for rotation about any axis perpendicular to the direction of the rod (proportional to its rotational constant) is the mean

11 E. U. Condon and G. H. Shortlex, The Theory of Atomic Spectra, The University Press, Cambridge 1957, p. 103.

12 D. I. Blochinzew, Grundlagen der Quantenmechanik, VEB Deutscher Verlag der Wissenschaften, Berlin 1963, p. 357.

13 S. FLüGGE, Lehrbuch der theoretischen Physik, Band IV, Quantentheorie I, Springer-Verlag, Berlin 1964, p. 341.

14 H. C. Allen and P. C. Cross, Molecular Vib-Rotors, J. Wiley and Sons, New York 1963, p. 106 and 261. $\frac{1}{2}\left(1 / I_{g^{\prime}}+1 / I_{g^{\prime \prime}}\right)$ of the molecule's reciprocal moments of inertia $1 / I_{g^{\prime}}$ and $1 / I_{g^{\prime \prime}}$; the moment of inertia for rotation about the direction of the rod vanishes. Working along the outline given in ref. ${ }^{11}$ one obtains the polarizability of this classical oscillator via the amplitude of the forced oscillation and the additional moment with frequency $v$ induced thereby as

$$
\begin{aligned}
& \alpha_{\mathrm{el}}^{g}\left(J^{\prime} \tau^{\prime}, J \tau\right) \\
& \quad=\mu_{g^{2}} \frac{1}{2}\left(\frac{1}{I_{g^{\prime}}}+\frac{1}{I_{g^{\prime \prime}}}\right) \cdot \frac{2}{3} \cdot \frac{1}{4 \pi^{2}\left(v^{2}\left(J^{\prime} \tau^{\prime}, J \tau\right)-v^{2}\right)}
\end{aligned}
$$

where the factor $2 / 3$ is the result of averaging over the arbitrary zero-direction of the vibrating rod with respect to the electrical vector of the light wave. It is now seen from (11) and (13) that (12) is indeed satisfied when the quantity $f^{g}\left(J^{\prime} \tau^{\prime}, J \tau\right)$ is taken to be the "rotator strength" as defined in (4).

\section{Conclusion}

The "partial $f$-sum rules for rotator strengths" derived in this paper as well as the (dependent) " $f$ sum rule for rotator strengths" given in paper I supplement the familiar sum rules over rotational energies and rotational line strengths ${ }^{14,15}$ alone. In addition to furnishing useful relations to check calculations or computer programs it has been demonstrated in these two papers how and with what modifications the original concept of "oscillator strengths" can be extended to the rotational transitions of an asymmetric top molecule with a permanent dipole moment interacting with the radiation field.

This work has been supported in part by the Deutsche Forschungsgemeinschaft and the Fonds der Chemischen Industrie.

15 Partial sum rules for line strengths alone follow easily from Eqs. (20) and (21) of ref. ${ }^{8}$, e. g.

$$
\begin{array}{r}
(2 J+1) \sum_{\tau^{\prime}} S g\left(J+1 \tau^{\prime}, J \tau\right)+J \sum_{\tau^{\prime}} S^{g}\left(J \tau^{\prime}, J \tau\right) \\
=(J+1)(2 J+1), \\
(J+1) \sum_{\tau^{\prime}} S g\left(J \tau^{\prime}, J \tau\right)+(2 J+1) \sum_{\tau^{\prime}} S g\left(J-1 \tau^{\prime}, J \tau\right) \\
=J(2 J+1) .
\end{array}
$$

